INPLASY

PROTOCOL

To cite: Xin et al. A metaanalysis of the accuracy of neutrophil to lymphocyte ratio in diagnosing neonatal sepsis. Inplasy protocol 202190043. doi:

10.37766/inplasy2021.9.0043

Received: 14 September 2021

Published: 14 September 2021

Corresponding author:

Xin yu

xy15774634247@163.com

Author Affiliation:

Department of Critical Care Medicine, Harbin Medical University Cancer Hospital, Harbin, China.

Support: No funding.

Review Stage at time of this submission: Other (Not specified).

Conflicts of interest:

None declared.

\section{A meta-analysis of the accuracy of neutrophil to lymphocyte ratio in diagnosing neonatal sepsis}

Xin, $Y^{1}$; Shao, $Y^{2}$.

Review question / Objective: What is the predictive value of neutrophil to lymphocyte ratio in diagnosing neonatal sepsis? Condition being studied: The incidence rate of neonatal sepsis is $1 \%-20 \%$, which is the third place after preterm birth and neonatal encephalopathy (asphyxia and trauma during childbirth). Due to the sensitivity of disease diagnosis methods and the timeliness and Effectiveness of the whole treatment process, the mortality rate of neonatal sepsis is increasing year by year.At present, the neutrophil to lymphocyte ratio (NLR) has been widely used as an independent predictor of various diseases in clinic, such as inflammatory indicators of immune system status, tumors, cancers, etc. The purpose of this study was to evaluate the predictive value of NLR in the diagnosis of neonatal sepsis, and to provide a basis for clinical diagnosis of neonatal sepsis.

INPLASY registration number: This protocol was registered with the International Platform of Registered Systematic Review and Meta-Analysis Protocols (INPLASY) on 14 September 2021 and was last updated on 14 September 2021 (registration number INPLASY202190043).

\section{INTRODUCTION}

Review question / Objective: What is the predictive value of neutrophil to lymphocyte ratio in diagnosing neonatal sepsis?
Condition being studied: The incidence rate of neonatal sepsis is $1 \%-20 \%$, which is the third place after preterm birth and neonatal encephalopathy (asphyxia and trauma during childbirth). Due to the sensitivity of 
disease diagnosis methods and the timeliness and Effectiveness of the whole treatment process, the mortality rate of neonatal sepsis is increasing year by year. At present, the neutrophil to lymphocyte ratio (NLR) has been widely used as an independent predictor of various diseases in clinic, such as inflammatory indicators of immune system status, tumors, cancers, etc. The purpose of this study was to evaluate the predictive value of NLR in the diagnosis of neonatal sepsis, and to provide a basis for clinical diagnosis of neonatal sepsis.

\section{METHODS}

Participant or population: The subjects were newborns with sepsis (Diagnosis by culture of menstrual blood).

Intervention: Neutrophil to lymphocyte ratio (NLR) were used for neonatal sepsis diagnosis.

Comparator: Health people served as the controls.

Study designs to be included: Prospective studies, cohort studies, case-control studies, or cross -sectional studies.

Eligibility criteria: Inclusion criteria: The purpose of this study was to evaluate or discuss the diagnostic value of neutrophil to lymphocyte ratio in neonatal sepsis; The experimental group was diagnosed with sepsis, and the control group was healthy. The gold standard of diagnosis was blood culture; The true positive value, false positive value, true negative value and false negative value of neutrophil lymphocyte ratio in diagnosis of neonatal sepsis can be obtained directly or indirectly; The language is English. Exclusion criteria: The true positive value, false positive value, true negative value and false negative value can not be directly extracted from the full text or calculated. Review, conference report, case and animal experiments; Repeated publication of literature.
Information sources: All studies were obtained by searching English databases:Cochrane, PubMed, Embase, Web of Science, medline. Keywords and free words are used to search. Search terms include:Neutrophil and lymphocyte ratio", "Infant", "Newborn", "Neonate", "sepsis", "septicemia", "Neonatal Sepsis".

Main outcome(s): Sensitivity; Specificity; Positive and negative likelihood ratios; under the curve and their respective 95\% confidence intervals.

Quality assessment / Risk of bias analysis: Quadas-2 was used to evaluate the quality of the included literature and the possibility of bias. If there is disagreement, resolve it through negotiation or discussion with a third investigator.

Strategy of data synthesis: Meta-disc1.4 software was used to analyze the heterogeneity of threshold effect, and 12 was used to evaluate the heterogeneity. If the effect sizes are homogenous, the fixed effect model is adopted. If heterogeneity is present, random effect model is used. If heterogeneity exists between studies, the source of heterogeneity will be further explored, and meta-regression analysis, threshold effect and non-threshold effect will be conducted. Combined sensitivity, combined specificity, combined diagnostic odds ratio (DOR), combined positive likelihood ratio (PLR), combined negative likelihood ratio (NLR) and $95 \%$ confidence interval $(95 \% \mathrm{Cl})$ were calculated by STATa16.0. At the same time, the combined receiver operating characteristic curve (SROC) fitting analysis was performed. At the same time, Deeks test was used to evaluate the publication bias of the included literatures. If $P<0.05$, the included literatures were considered to have publication bias.

Subgroup analysis: Subgroup analysis include elary onset sepsis; cutoff2.

Sensitivity analysis: This study will perform a sensitivity analysis to examine the stability of study findings by excluding low quality study. 
Country(ies) involved: China.

Keywords: sepsis; neonatal; diagnosis;

Neutrophil to lymphocyte ratio; Meta analysis.

Contributions of each author:

Author 1 - Xin yu.

Author 2 - Shao yunshuang. 